Classification

Physics Abstracts

$05.20-05.40-66.90$

\title{
On the flux distribution in a one dimensional disordered system
}

\author{
Cécile Monthus $\left({ }^{1}\right)$ and Alain Comtet $\left({ }^{2}\right)$ \\ $\left({ }^{1}\right)$ Division de Physique Théorique( ${ }^{*}$ ), IPN, 91406 Orsay Cedex,France \\ $\left({ }^{2}\right)$ LPTPE, Université Pierre et Marie Curie, 4 Place Jussieu, 75252 Paris Cedex 05, France
}

(Received 10 December 1993, accepted 10 February 1994)

\begin{abstract}
Résumé . - Nous étudions quelques propriétés de transport d'un système unidimensionnel désordonné de longueur finie $N$. Dans ce système, les particules sont soumises à des forces aléatoires quı résultent à la fois d'un bruit d'origine thermique et d'une force aléatoire gelée $F(x)$ qui caractérıse le fait que l'environnement est inhomogène. On suppose que cette force est distribuée comme un bruit blanc avec une valeur moyenne non nulle. En présence d'une concentration finie de particules aux deux extrémités de la chaîne, ll apparart un courant de diffusion stationnaire $J(N)$ qui dépend de l'environnement $\{F(x)\}$. Nous étudions la distribution de probabilité $P(J)$ de ce courant. Notre approche s'appuie à la fois sur une méthode fonctionnelle et sur un calcul de moments. Dans le cas d'un biais non nul, nos résultats constituent une généralisation de ceux obtenus récemment par Oshanin et al.
\end{abstract}

\begin{abstract}
We study some transport properties of a one dimensional disordered system of finite length $N$. In this system particles are subject to random forces resulting both from a thermal noise and from a quenched random force $F(x)$ which models the inhomogeneous medium. The latter is distributed as a white noise with a non zero average bias. Imposing some fixed concentration of particles at the end points of the chain yields a steady current $J(N)$ which depends on the environment $\{F(x)\}$. The problem of computing the probability distribution $P(J)$ over the environments is addressed. Our approach is based on a path integral method and on a moment calculation. In the case of a non zero bias our results generalize those obtained recently by Oshanin et al.
\end{abstract}

\section{Introduction.}

A large amount of work has been devoted to the study of classical diffusion in random media. Part of the interest in this field comes from the fact that the transport properties in such systems are very different from those of homogeneous media. In particular it has been shown that quite generically one expects an anomalous diffusion behaviour to occur [1].

$\left({ }^{*}\right)$ Unité de Recherche des Universités Parıs 11 et Parıs 6 Associée au CNRS. 
The simplest one dimensional model which gives rise to such anomalous behaviour is known as the Sinai model [2]. In this model, one considers a one dimensional lattice on which, at integer times, a particle can jump from site $n$ to site $n+1$ with probability $p_{n}$, or from site $n$ to site $n-1$ with probability $q_{n}=1-p_{n}$. The $p_{n}(n \in Z)$ is a set of independent random variables distributed according to some probability law $\rho(p)$ such that

$$
\begin{aligned}
& <\log \frac{p_{3}}{1-p_{3}}>=\int \rho(p) \log \frac{p}{1-p} \mathrm{~d} p=0 \\
& <\log ^{2} \frac{p_{3}}{1-p_{3}}>=\int \rho(p) \log ^{2} \frac{p}{1-p} \mathrm{~d} p<\infty .
\end{aligned}
$$

In this case it has been proved that the mean square displacement $\left\langle x^{2}(t)\right\rangle$ grows like $(\log t)^{4}$ for $t \rightarrow \infty$.

This problem can also be studied by starting from a continuous model defined by the Langevin equation

$$
\dot{x}=\frac{1}{\gamma} F(x)+\eta(t)
$$

where $F(x)$ is a quenched random force (related to the hopping variables $p_{\jmath}$ ) which is usually taken as a Gaussian white noise

$$
\begin{aligned}
& <F(x)>=F_{\mathrm{o}} \\
& <F(x) F\left(x^{\prime}\right)>-<F(x)>^{2}=\sigma \delta\left(x-x^{\prime}\right)
\end{aligned}
$$

and $\eta(t)$ is a thermal noise randomly chosen at each time such that

$$
\begin{aligned}
& \overline{\eta(t)}=0 \\
& \overline{\eta(t) \eta\left(t^{\prime}\right)}=\frac{2 k T}{\gamma} \delta\left(t-t^{\prime}\right) .
\end{aligned}
$$

The Sinai case corresponds to an average bias $F_{\mathrm{o}}=0$. Increasing $F_{\mathrm{o}}$, one obtains a succession of phases [3, 4] which in terms of $\mu=\frac{2 k T}{\sigma} F_{\text {o }}$ read

1) $0<\mu<1$ : anomalous dispersion $\left\langle x(t)>\sim t^{\mu}\right.$.

2) $1<\mu<2$ : one obtains a finite velocity, $V=\frac{F_{\mathrm{o}}}{\gamma}\left(1-\frac{1}{\mu}\right)$, but the diffusion remains anomalous

$$
<x(t)>-V t \sim t^{\frac{3-\mu}{2}}
$$

3) $\mu>2$, one recovers a normal diffusion with a finite diffusion constant

$$
D=D_{\circ} \frac{\mu-1}{\mu-2}
$$

where $D_{\mathrm{o}}=k T / \gamma=1 /(\beta \gamma)$ is the diffusion constant of the pure system.

A quantitative understanding of these phases has been obtained by Bouchaud et al. [4]. For a non zero bias $F_{\mathrm{o}}$, it was conjectured that the diffusion process is essentially controlled by the existence of very high potential barriers that the particle has to overcome by thermal activation. Assuming that these barriers act as trapping regions between which the motion is purely convective, one is thus led to a simpler picture in which the problem becomes essentially equivalent at large time to a directed walk among traps. 
The sojourn time $T(x)$, which measures the thermal average of the time spent at position $x$ was identified as

$$
T(x)=\int_{x}^{\infty} \mathrm{d} \xi \exp -\beta \int_{x}^{\xi} F(z) \mathrm{d} z .
$$

In suitable units this random variable is distributed over the environments $\{F(x)\}$ according to the law [4]

$$
\psi(T)=\frac{1}{\Gamma(\mu) T^{\mu+1}} \mathrm{e}^{-1 / T}
$$

Similar random variables have been studied in the mathematical [5] as well as in the physical $[6,7]$ literature. The occurrence of a power law decay for large $T$ seems to be a rather general feature, which is related to the anomalous behavior of $x(t)$ through a Tauberian theorem [8].

A similar variable, with however a different physical interpretation, also appears in the study of transport properties of the Sinai model [9]. Consider a finite chain of length $N$ with some prescribed concentration of particles at the end points

$$
\begin{aligned}
& P(x=0, t)=P_{0} \\
& P(x=N, t)=P_{N} .
\end{aligned}
$$

In such a system, for a given environment $\{F(x)\}$, there exists a steady current

$$
J(N)=\left[-D_{0} \frac{\mathrm{d} P}{\mathrm{~d} x}+\frac{F(x)}{\gamma} P(x)\right] .
$$

Taking into account the boundary conditions (1.9), it is given by

$$
J(N)=\frac{D_{0} P_{0}}{\tau(N)}-D_{0} P_{N} \frac{\partial}{\partial N} \ln \tau(N)
$$

where

$$
\tau(N)=\int_{0}^{N} \mathrm{~d} \xi \exp -\beta \int_{0}^{\xi} \mathrm{d} \xi F(\xi) .
$$

In the Sinai case $\langle F(x)\rangle=0$, it has been shown that the disorder average flux $\langle J(N)\rangle$ has a non Fickian behaviour, namely

$$
<J(N)>\underset{N \rightarrow \infty}{\sim} \frac{1}{\sqrt{N}} .
$$

This result was first obtained by a careful estimation of upper and lower bounds of the current [10]. More precise results have been recently obtained, in particular the whole distribution $\psi(\tau)$ of the random variable $\tau$ (or alternatively of $J$ for the case $P_{N}=0$ ) has been obtained [9]. The method is based on the evaluation of the moments $\left\langle\tau^{n}\right\rangle$ and on a suitable resummation of the series giving the generating function

$$
\left\langle\mathrm{e}^{-p \tau}\right\rangle=\sum_{n=0}^{\infty} \frac{(-p)^{n}}{n !}<\tau^{n}>
$$

The purpose of this work is to present an alternative derivation of these results by a path integral technique. A generalisation to the case of a non zero bias is also presented. We will consider the cases $\mu>0$ and $\mu<0$ since they correspond to different physical situations due to the choice of the boundary conditions. 


\section{The Sinai case.}

In this section we study the distribution of the random variable

$$
\tau(N)=\int_{0}^{N} \mathrm{~d} \xi \exp -\beta \int_{0}^{\xi} \mathrm{d} \xi^{\prime} F\left(\xi^{\prime}\right)
$$

when the average bias $F_{0}$ vanishes. Averaging over the random force, we can construct the generating function

$$
\begin{aligned}
\phi(p, N) & =\langle\exp -[p r(N)]> \\
& =\int \mathcal{D} F(x) \exp -\left[\frac{1}{2 \sigma} \int_{0}^{N} \mathrm{~d} \xi F^{2}(\xi)+p \int_{0}^{N} \mathrm{~d} \xi \exp -\beta \int_{0}^{\xi} \mathrm{d} \xi^{\prime} F\left(\xi^{\prime}\right)\right]
\end{aligned}
$$

From the generating function one can derive the probability distribution $\psi(\tau, N)$

$$
\phi(p, N)=\int_{0}^{\infty} \mathrm{e}^{-p \tau} \psi(\tau, N) \mathrm{d} \tau
$$

It is convenient to rewrite equation (2.2) in terms of the potential

$$
U(\xi)=-\int_{0}^{\xi} F\left(\xi^{\prime}\right) \mathrm{d} \xi
$$

One obtains

$$
\phi(p, N)=\int_{U(0)=0} \mathcal{D} U(\xi) \exp -\frac{1}{2 \sigma} \int_{0}^{N} \mathrm{~d} \xi\left(\frac{\mathrm{d} U}{\mathrm{~d} \xi}\right)^{2}-p \int_{0}^{N} \mathrm{~d} \xi \exp +\beta U(\xi) .
$$

This path integral describes the motion of a particle of mass $1 / \sigma$ moving in the potential

$$
V(U)=p \exp \beta U
$$

The associated Hamiltonian reads

$$
H=-\frac{\sigma}{2} \frac{\mathrm{d}^{2}}{\mathrm{~d} U^{2}}+p \exp \beta U
$$

Let us denote by $\psi_{k}(U)$ a complete set of eigenstates of $H$ such that

$$
H \psi_{k}(U)=k^{2} \psi_{k}(U)
$$

The generating function then reads

$$
\phi(p, N)=\int_{-\infty}^{\infty} \mathrm{d} k \int_{-\infty}^{\infty} \mathrm{d} U \psi_{k}(U) \psi_{k}^{*}(0) \mathrm{e}^{-k^{2} N}
$$

The solutions of (2.8) which vanish at $U=+\infty$ are

$$
\psi_{k}(U)=N K_{2 i k / \sqrt{\alpha}}\left(2 \sqrt{\frac{p}{\alpha}} \mathrm{e}^{\beta U / 2}\right)
$$


where $N$ is a normalization constant which is fixed by the continuum normalization

$$
\int_{-\infty}^{+\infty} \psi_{k}(U) \psi_{q}^{*}(U) \mathrm{d} U=\delta(k-q)
$$

and $1 / \alpha=2 /\left(\sigma \beta^{2}\right)$ is a surtable unit of length.

One obtains

$$
\psi_{k}(U)=2 \sqrt{\frac{\beta k}{\alpha \pi} \sinh \frac{2 k \pi}{\sqrt{\alpha}}} K_{2 \imath k / \sqrt{\alpha}}\left(2 \sqrt{\frac{p}{\alpha}} \mathrm{e}^{\beta U / 2}\right) .
$$

Carrying out the integral over $U$ in equation (2.9) yields the final expression

$$
\phi(p, N)=\frac{2}{\pi} \int_{0}^{\infty} \cosh \frac{\pi k}{2} K_{i k}\left(2 \sqrt{\frac{p}{\alpha}}\right) \mathrm{e}^{-k^{2} \alpha N / 4} \mathrm{~d} k
$$

which is in agreement with equation (16) of [9].

From this expression one can study the behaviour of the probability law $\psi(\tau, N)$ in the limit in which the size of the sample is very large

$$
\phi(p, N) \underset{N \rightarrow \infty}{\sim} \frac{2}{\sqrt{\alpha \pi N}} K_{0}\left(2 \sqrt{\frac{p}{\alpha}}\right) .
$$

For fixed $\tau$ the probability law therefore reads

$$
\psi(\tau, N) \underset{N \rightarrow \infty}{\sim} \frac{1}{\sqrt{\alpha \pi N}} \frac{1}{\tau} \exp -\frac{1}{\alpha \tau} .
$$

Since this result does not hold uniformly with respect to $\tau$ this distribution is obviously not normalized. The true behaviour for large $\tau$ is in fact given by the log-normal tail

$$
\psi(\tau, N) \underset{\tau \rightarrow \infty}{\sim} \frac{1}{2 \sqrt{\alpha \pi N}} \frac{1}{\tau} \exp \left[-\frac{1}{4 \alpha N} \ln ^{2}(\alpha \tau)\right] .
$$

It is however interesting to point out that equation (2.15) that characterizes the tail of the distribution for $\tau \rightarrow 0$ is in fact consistent with equation (1.8) in the limit $\mu \rightarrow 0$.

\section{Functional integration method for the case $\langle F\rangle \neq 0$.}

The quenched random force $\{F(x)\}$ is now a Gaussian white noise with a non-zero mean

$$
\begin{aligned}
& <F(x)>=F_{0} \\
& <F(x) F\left(x^{\prime}\right)>-F_{0}^{2}=\sigma \delta\left(x-x^{\prime}\right) .
\end{aligned}
$$

The generating function for $\tau(N)=\int_{0}^{N} \mathrm{~d} x \mathrm{e}^{-\beta \int_{0}^{x} F(y) \mathrm{d} y}$ reads

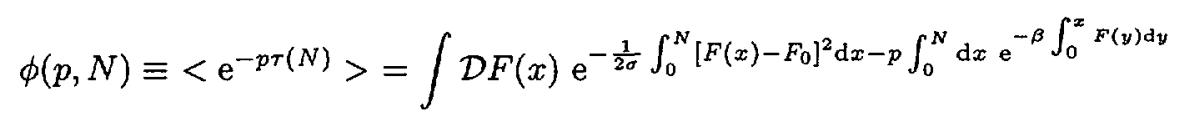


As in the case $\mu=0$, we rewrite this path integral over the force $\{F(x)\}$ as a path integral over the potential $U(x)=-\int_{0}^{x} F(y) \mathrm{d} y$

$$
\begin{aligned}
\phi(p, N) & =\int_{U(0)=0} \mathcal{D} U(x) \mathrm{e}^{-\frac{1}{2 \sigma} \int_{0}^{N}\left(\frac{\mathrm{d} U}{\mathrm{~d} x}+F_{0}\right)^{2} \mathrm{~d} x-p \int_{0}^{N} \mathrm{~d} x \mathrm{e}^{\beta U(x)}} \\
& =\mathrm{e}^{-\frac{F_{0}^{2} N}{2 \sigma}} \int_{-\infty}^{+\infty} \mathrm{d} u \mathrm{e}^{-\frac{F_{0}}{\sigma} u} \quad\left\langle u\left|\mathrm{e}^{-N H}\right| 0\right\rangle
\end{aligned}
$$

where $\left\langle u\left|\mathrm{e}^{-N H}\right| 0\right\rangle$ is the Green function that we have already encountered in the case $\mu=0$. Its expansion in terms of a complete set of eigenstates $\left(\psi_{k}\right)$ of $H$ gives

$$
\begin{aligned}
\left.<u\left|\mathrm{e}^{-N H}\right| 0\right\rangle & =\int_{U(0)=0}^{U(N)=u} \mathcal{D} U(x) \mathrm{e}^{-\int_{0}^{N} \mathrm{~d} x\left[\frac{1}{2 \sigma}\left(\frac{\mathrm{d} U}{\mathrm{~d} x}\right)^{2}+p \mathrm{e}^{\beta U(x)}\right]} \\
& =\int_{-\infty}^{+\infty} \mathrm{d} k \psi_{k}^{*}(0) \psi_{k}(u) \mathrm{e}^{-k^{2} N}
\end{aligned}
$$

From the explicit expression of $\psi_{k}(u)$ one obtains through the change of variables

$$
\begin{aligned}
& \left\{\begin{array}{l}
q=\frac{2}{\sqrt{\alpha}} k \\
x=\sqrt{\frac{p}{\alpha}} \mathrm{e}^{\beta u / 2}
\end{array}\right. \\
& \phi(p, N)=\frac{\mathrm{e}^{-\frac{\alpha N}{4} \mu^{2}}}{\pi^{2}}\left(\frac{p}{\alpha}\right)^{\mu} \int_{0}^{\infty} \frac{\mathrm{d} x}{x^{1+\mu}} \int_{-\infty}^{+\infty} \mathrm{d} q \mathrm{e}^{-\frac{\alpha N}{4} q^{2}} q \sinh \pi q K_{2 q}(2 x) K_{2 q}\left(2 \sqrt{\frac{p}{\alpha}}\right)
\end{aligned}
$$

where $\mu=\frac{2 F_{0}}{\sigma \beta}$ is the dimensionless parameter that characterizes the various phases of the problem. (See Ref. [4] for a physical discussion of this parameter).

However, this result, as it stands, is not fully satisfactory for two reasons. First, one cannot expand the expression of $\phi(p, N)$ into an entire series in $p$ in order to extract the moments of $\tau(N)$. Furthermore it is very difficult to study the limit $N \rightarrow \infty$ when $\mu>0$.

Let us first consider the case $\mu<0$.

In this case interchanging the two integrations, one obtains

$\phi(p, N)=\frac{\mathrm{e}^{-\frac{\alpha N}{4} \mu^{2}}}{4 \pi^{2}}\left(\frac{p}{\alpha}\right)^{\mu / 2} \int_{-\infty}^{+\infty} \mathrm{d} q \mathrm{e}^{-\frac{\alpha N}{4} q^{2}} q \sinh \pi q\left|\Gamma\left(-\frac{\mu}{2}+\frac{i q}{2}\right)\right|^{2} K_{\imath q}\left(2 \sqrt{\frac{p}{\alpha}}\right)$.

From which it follows that there is no asymptotic law in the limit $N \rightarrow \infty$. In the case $\mu>0$, since the interchange of integrations is not allowed, we will use another approach which will be the subject of the next sections. First we will compute directly the moments $\left\langle\tau^{n}(N)\right\rangle$ for $n \in N$. Besides the fact that these quantities are interesting in themselves, they will enable us to prove that there is an asymptotic law for $\mu>0$ in the limit $N \rightarrow \infty$ and to find the expression of this law.

Then we will use these moments to reconstruct the characteristic function. For $\mu<0$ we will recover the above expression (3.6), but for $\mu>0$ we will find a more tractable formula than (3.5). This will allow us to study how the full distribution $\psi(\tau, N)$ approaches the asymptotic law $\psi(\tau)$ in the limit $N \rightarrow \infty$. 


\section{Moments.}

We now turn to the derivation of the moments of the random variable $\tau(N)$. The moment of order $n$ reads by definition

$$
<\tau^{n}(N)>=\int \mathcal{D} F(x) \mathrm{e}^{-\frac{1}{2 \sigma} \int_{-\infty}^{+\infty}\left[F(x)-F_{0}\right]^{2} \mathrm{~d} x} \prod_{i=1}^{n}\left(\int_{0}^{N} \mathrm{~d} x_{i} \mathrm{e}^{-\beta \int_{0}^{x_{i}} F(x) \mathrm{d} x}\right)
$$

Performing the Gaussian functional integral over the random force $F(x)$ we are left with an ordinary integral over $\mathrm{n}$ varıables

$$
<\tau^{n}(N)>=n ! \int_{0 \leq x_{1} \leq x_{2} \cdots \leq x_{n} \leq N}\left(\prod_{\imath=1}^{n} \mathrm{~d} x_{\imath}\right) \mathrm{e}^{\alpha \sum_{k=1}^{n} x_{\ell}[1+2(n-k)-\mu]} .
$$

For $\mu \notin N^{*}$, we get

$$
<\alpha^{n} \tau^{n}(N)>=\sum_{k=0}^{n} \mathrm{e}^{\alpha N k(k-\mu)}(-1)^{n-k} C_{n}^{k}\left(\prod_{i=1}^{n-k} \frac{1}{2 k-\mu+i}\right)\left(\prod_{j=1}^{k} \frac{1}{k-\mu-1+j}\right)
$$

- In the case $\mu=0$, we recover the result (Oshanin et al.)

$$
<\alpha^{n} \tau^{n}(N)>=\sum_{k=1}^{n} \mathrm{e}^{\alpha N k^{2}}(-1)^{n-k} \frac{\Gamma(n)}{\Gamma(2 n)} C_{2 n}^{k}+\frac{(-1)^{n}}{n !}
$$

- $\quad$ For $\mu \notin N^{*}$ making use of the basic property of $\Gamma$ function $[\Gamma(z+1)=z \Gamma(z)]$

$$
<\alpha^{n} \tau^{n}(N)>=\sum_{k=0}^{n} \mathrm{e}^{\alpha N k(k-\mu)}(-1)^{n-k} C_{n}^{k}(2 k-\mu) \frac{\Gamma(k-\mu)}{\Gamma(n+1+k-\mu)} .
$$

We now consider the limit $N \rightarrow \infty$ in the case $\mu>0$.

From (4.5) one finds

$$
<\alpha^{n} \tau^{n}(N)>\underset{N \rightarrow \infty}{\rightarrow}\left\{\begin{array}{cl}
\infty & \text { if } \quad \mu<n \\
\frac{\Gamma(\mu-n)}{\Gamma(\mu)} & \text { if } \quad \mu>n
\end{array}\right.
$$

The asymptotic moments can thus be rewritten as

$$
<\alpha^{n} \tau^{n}(\infty)>=\frac{1}{\Gamma(\mu)} \int_{0}^{\infty} \mathrm{d} t t^{\mu-n-1} \mathrm{e}^{-t} \mathrm{~d} t
$$

where we have used the integral representation of $\Gamma$ function for positive argument.

The change of variables $t=1 /(\alpha \tau)$ gives the asymptotic probability density of $\tau(N)$ in the limit $N \rightarrow \infty$

$$
\psi(\tau, N) \underset{N \rightarrow \infty}{\rightarrow} \psi_{\infty}(\tau)=\frac{\alpha}{\Gamma(\mu)}\left(\frac{1}{\alpha \tau}\right)^{\mu+1} \mathrm{e}^{-\frac{1}{\alpha \tau}}
$$


The corresponding generating function therefore reads

$$
\begin{aligned}
<\mathrm{e}^{-p \tau(\infty)}> & =\int_{0}^{\infty} \mathrm{d} \tau \mathrm{e}^{-p \tau} \psi_{\infty}(\tau) \\
& =\frac{2}{\Gamma(\mu)}\left(\frac{p}{\alpha}\right)^{\mu / 2} K_{\mu}\left(2 \sqrt{\frac{p}{\alpha}}\right) \\
& =\Gamma(1-\mu) \sum_{l=0}^{\infty} \frac{1}{l !}\left[\frac{\left(\frac{p}{\alpha}\right)^{l}}{\Gamma(1+l-\mu)}-\frac{\left(\frac{p}{\alpha}\right)^{l+\mu}}{\Gamma(1+l+\mu)}\right]
\end{aligned}
$$

It is interesting to note that the first non integer power in this expansion is $(p / \alpha)^{\mu}$. This implies that the derivative of order $n$ at $p=0$ is finite for $n<\mu$ and infinite for $n>\mu$. This shows explicitly at the level of the generating function why only the moments with $n<\mu$ are finite.

\section{Construction of the generating function from the moments.}

For a finite chain of length $N$, all the moments of $\tau(N)$ are finite, however we cannot directly construct the generating function from the entire series $\left\langle e^{-p \tau(N)}\right\rangle=\sum_{n=0}^{\infty} \frac{(-p)^{n}}{n !}$ $\left.<\tau^{n}(N)\right\rangle$ since its convergence radius vanishes.

Indeed, using equation (4.5), we see that the general term of this series tends to infinity for all $p \neq 0$

$$
\left|\frac{p^{n}}{n !}<\tau^{n}(N)>\right| \underset{n \rightarrow \infty}{\sim}\left|\frac{p^{n}}{\Gamma(n+1)} \frac{\Gamma(n-\mu)}{\Gamma(2 n-\mu)} \mathrm{e}^{\alpha N n(n-\mu)}\right|_{n \rightarrow \infty}^{\rightarrow} \infty .
$$

In order to derive the true generating function, we therefore have to give a sensible meaning to this formal series, forgetting that we are in fact in a case where the knowledge of the moments does not uniquely determine the probability distribution [11]. This will now be done by two methods.

5.1 FirSt METHOD : DIFFERENTIAL EQUATION FOR $\phi(p, N)$. - From (4.5) it follows that the derivative of the moment of order $n$ with respect to $N$ is a simple combination of moments of order $n$ and $(n-1)$

$$
\frac{1}{\alpha} \frac{\partial}{\partial N}<\alpha^{n} \tau^{n}(N)>=n(n-\mu)<\alpha^{n} \tau^{n}(N)>+n<\alpha^{n-1} \tau^{n-1}(N)>
$$

This recurrence relation induces a differential equation for the formal series

$$
\begin{aligned}
& \phi(p, N)=\sum_{n=0}^{\infty} \frac{(-p)^{n}}{n !}<\tau^{n}(N)> \\
& \qquad \frac{1}{\alpha} \frac{\partial \phi}{\partial N}=p^{2} \frac{\partial^{2} \phi}{\partial p^{2}}+(1-\mu) p \frac{\partial \phi}{\partial p}-\frac{p}{\alpha} \phi
\end{aligned}
$$

which has to be supplemented with the initial condition at $N=0: \phi(p, 0)=1$ since we have by definition $\tau(N=0)=0$. 
We can thus write the corresponding differential equation for the probability density $\psi(\tau, N)$, which is just the inverse Laplace transform of $\phi(p, N)$

$$
\frac{1}{\alpha} \frac{\partial \psi}{\partial N}=\frac{\partial}{\partial \tau}\left[\tau^{2} \frac{\partial \psi}{\partial \tau}+\left[(\mu+1) \tau-\frac{1}{\alpha}\right] \psi\right] .
$$

The initial condition at $N=0$ now reads : $\psi(\tau, 0)=\delta(\tau)$. It is straightforward to recover the asymptotic law $\psi_{\infty}(\tau)$ (Eq. (4.6)) as the stationary solution of this equation $(\mu>0)$. In order to obtain the general solution it is convenient to perform the change of variable $\alpha \tau=2 / x^{2}$.

The corresponding probability density $P(x, N)$ such that $P(x, N)|\mathrm{d} x|=\Psi(\tau, N)|\mathrm{d} \tau|$ satisfies

$$
\frac{4}{\alpha} \frac{\partial P}{\partial N}=\frac{\partial^{2}}{\partial x^{2}}\left(x^{2} P\right)-\frac{\partial}{\partial x}\left[(2 \mu+1) x-x^{3}\right] P \text {. }
$$

It turns out that this equation has been studied in great detail by Schenzle et al., as a model of a multiplicative stochastic process [12]. The general solution can be expressed in terms of an eigenfunction expansion which consists of a discrete and a continuous branch. New discrete states are formed when one increases the drift $\mu$ (which in their model plays the role a pump parameter). Using these results (see appendix A) supplemented by the initial condition at $N=0$ gives the probability density

$$
\begin{aligned}
& \psi(\tau, N)=\sum_{0 \leq n<\frac{\mu}{2}} \mathrm{e}^{-\alpha N n(\mu-n)} \frac{(-1)^{n}(\mu-2 n)}{\Gamma(1+\mu-n)}\left(\frac{1}{\alpha \tau}\right)^{1+\mu-n} L_{n}^{\mu-2 n}\left(\frac{1}{\alpha \tau}\right) \mathrm{e}^{-\frac{1}{\alpha \tau}} \\
& +\frac{\alpha}{4 \pi^{2}} \int_{0}^{\infty} \mathrm{d} s \mathrm{e}^{-\frac{\alpha N}{4}\left(\mu^{2}+s^{2}\right)} s \sinh \pi s\left|\Gamma\left(-\frac{\mu}{2}+i \frac{s}{2}\right)\right|^{2}\left(\frac{1}{\alpha \tau}\right)^{\frac{1+\mu}{2}} W_{\frac{1+\mu}{2}, i \frac{s}{2}}\left(\frac{1}{\alpha \tau}\right) \mathrm{e}^{-\frac{1}{2 \alpha \tau}}
\end{aligned}
$$

where $L_{n}^{\alpha}$ are Laguerre's polynomials and $W_{p, \nu}$ are Whittaker's functions.

Laplace transformation of equation (5.5) gives the generating function

$$
\begin{aligned}
& \phi(p, N)=\sum_{0 \leq n<\frac{\mu}{2}} \mathrm{e}^{-\alpha N n(\mu-n)} \frac{2(\mu-2 n)}{n ! \Gamma(1+\mu-n)}\left(\frac{p}{\alpha}\right)^{\mu / 2} K_{\mu-2 n}\left(2 \sqrt{\frac{p}{\alpha}}\right) \\
& +\frac{1}{2 \pi^{2}} \int_{0}^{\infty} \mathrm{d} s \mathrm{e}^{-\frac{\alpha N}{4}\left(\mu^{2}+s^{2}\right)} s \sinh \pi s\left|\Gamma\left(-\frac{\mu}{2}+i \frac{s}{2}\right)\right|^{2}\left(\frac{p}{\alpha}\right)^{\mu / 2} K_{2 s}\left(2 \sqrt{\frac{p}{\alpha}}\right)
\end{aligned}
$$

Let us now discuss these results according to the value of the parameter $\mu$.

* For $\mu \leq 0$ we recover the results given by the functional method equation (3.6). The Fokker-Planck spectrum is purely continuous and, as a consequence, there is no asymptotic law in the limit $N \rightarrow \infty$.

* For $\mu>0$ we have now a more explicit expression than that given by the functional method (Eq. (3.5)). Besides the continuous branch, the Fokker-Planck spectrum also contains some discrete states. The ground state $n=0$ yields the asymptotic law in the limit $N \rightarrow \infty$ equation (4.7).

- For $0<\mu<2$, since the ground state is the only discrete state, the relaxation towards the asymptotic law is therefore given by the continuous branch.

- For $\mu>2$, it is the discrete state $n=1$ that governs the exponential relaxation towards the asymptotic law. 
5.2 SECOND METHOD : RESUMMATION OF THE FORMAL SERIES $\sum(-1)^{n} \frac{p^{n}}{n !}<\tau^{n}(N)>$. - Following Graham et al. [10], we now present another method of resummation based on a contour integral representation of the series.

As we have already seen at the beginning of this section, it is the dominant behaviour $\tau^{n}(N) \underset{n \rightarrow \infty}{\sim} \mathrm{e}^{\alpha N n^{2}}$ that makes the series $\sum(-1)^{n} \frac{p^{n}}{n !}<\tau^{n}(N)>$ divergent for $p \neq 0$. To regularize it, we insert the Gaussian integral identity

$$
\mathrm{e}^{\alpha N k(k-\mu)}=\frac{\mathrm{e}^{-\frac{\alpha N}{4} \mu^{2}}}{\sqrt{\pi}} \int_{-\infty}^{+\infty} \mathrm{d} x \mathrm{e}^{-x^{2}+(2 k-\mu) \sqrt{\alpha N} x}
$$

into equation (4.5) for the moment of order $n$

$$
\begin{aligned}
<\alpha^{n} \tau^{n}(N)>= & \frac{\mathrm{e}^{-\frac{\alpha N}{4} \mu^{2}}}{\sqrt{\pi}} \int_{-\infty}^{+\infty} \mathrm{d} x \mathrm{e}^{-x^{2}} \sum_{k=0}^{n} \mathrm{e}^{(2 k-\mu) \sqrt{\alpha N} x} \times \\
& \times(-1)^{n-k}(2 k-\mu) C_{n}^{k} \frac{\Gamma(k-\mu)}{\Gamma(n+1+k-\mu)} .
\end{aligned}
$$

Next we interchange the order of the integration and the sums. This gives a well-defined representation of the generating function

$$
\begin{aligned}
\phi(p, N) & =" \sum_{n=0}^{\infty} \frac{\left(-\frac{p}{\alpha}\right)^{n}}{n !}\left\langle\alpha^{n} \tau^{n}(N)>"\right. \\
& =\frac{\mathrm{e}^{-\frac{\alpha N}{4} \mu^{2}}}{\sqrt{\pi}}\left(\frac{p}{\alpha}\right)^{\mu / 2} \int_{-\infty}^{+\infty} \mathrm{d} x \mathrm{e}^{-x^{2}} \sum_{k=0}^{\infty} u_{k}(x)
\end{aligned}
$$

where

$$
u_{k}(x)=\mathrm{e}^{(2 k-\mu) \sqrt{\alpha N} x} \frac{(-1)^{k}}{k !}(2 k-\mu) \Gamma(k-\mu) I_{2 k-\mu}\left(2 \sqrt{\frac{p}{\alpha}}\right)
$$

The series $\sum_{k=0}^{\infty} u_{k}(x)$ is absolutely convergent for all $x$ according to d'Alembert's criterion : $\left|\frac{u_{k+1}(x)}{u_{k}(x)}\right|_{k \rightarrow \infty} 0$ since

$$
I_{M}(2 a)=\sum_{m=0}^{\infty} \frac{a^{2 m+M}}{m ! \Gamma(m+1+M)} \underset{M \rightarrow \infty}{\sim} \frac{a^{M}}{\Gamma(1+M)}
$$

We now introduce the function of the complex variable $s$

$$
f(s)=\mathrm{e}^{(2 s-\mu) \sqrt{\alpha N} x}(2 s-\mu) \Gamma(-s) \Gamma(s-\mu) I_{2 s-\mu}\left(2 \sqrt{\frac{p}{\alpha}}\right)
$$

$f$ admits two series of simples poles $(\mu \notin N)$ :

$\Gamma(-s)$ has poles at $s=k$, for $k \in N$, and $\operatorname{Res}(\Gamma(-s) ; k)=-\frac{(-1)^{k}}{k !}$ 
$\Gamma(s-\mu)$ has poles at $s=\mu-m$, for $m \in N$, and $\operatorname{Res}(\Gamma(s-\mu) ; \mu-m)=\frac{(-1)^{m}}{m !}$

Using Cauchy's theorem we can rewrite the series $\sum u_{k}$ as a contour integral in the complex $s$ plane

$$
\sum_{k=0}^{\infty} u_{k}=-\sum_{k=0}^{\infty} \operatorname{Res}(f ; k)=\frac{1}{2 \pi i} \int_{\mathcal{C}} f(s) \mathrm{d} s
$$

where $(\mathcal{C})$ is a negative oriented contour of the complex plane such that the poles of $\Gamma(-s)$ are inside and the poles of $\Gamma(\mu-s)$ are outside.

We have to distinguish the cases $\mu<0$ and $\mu>0$ and choose an explicit contour $(\mathcal{C})$ for each case.

a) case $\mu<0$

We choose the contour $\left(\mathcal{C}_{1}\right)$ drawn in figure 1 . The integral along the semicircle at infinity vanishes. To compute the remaining integral of $f$ along the line $\operatorname{Re}(s)=\mu / 2$ it is convenient to set $s=\mu / 2+i q / 2$, then

$$
\begin{aligned}
\sum_{k=0}^{\infty} u_{k}(x) & =\frac{1}{2 \pi i} \int_{\frac{\mu}{2}-\imath \infty}^{\frac{\mu}{2}+\imath \infty} f(s) \mathrm{d} s \\
& =\frac{i}{4 \pi} \int_{-\infty}^{+\infty} \mathrm{d} q q \mathrm{e}^{\imath q \sqrt{\alpha N} x}\left|\Gamma\left(-\frac{\mu}{2}+i \frac{q}{2}\right)\right|^{2} I_{2 q}\left(2 \sqrt{\frac{p}{\alpha}}\right)
\end{aligned}
$$

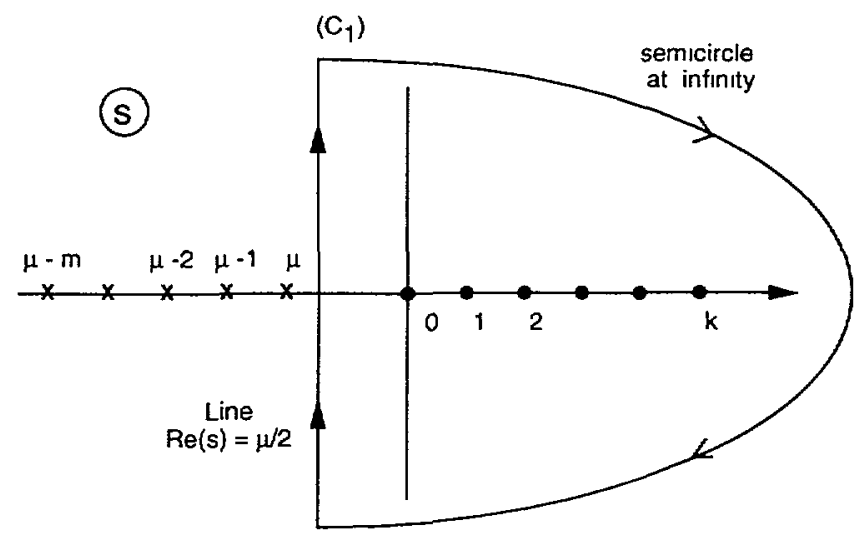

Fig. 1. - Contour $\mathrm{C}_{1}$ for the case $\mu<0$. Full crrcles that represent poles of $\Gamma(-s)$ at $s=k, k \in N$, are encirled once. Crosses that represent poles of $\Gamma(s-\mu)$ at $s=\mu-m, m \in N$, are not encircled.

Interchanging the integrations over the variables $x$ and $q$ we recover equation (5.6) in the case $\mu<0$

$$
\phi(p, N)=\frac{\mathrm{e}^{-\frac{\alpha N}{4} \mu^{2}}}{2 \pi^{2}} \int_{0}^{\infty} \mathrm{d} q \mathrm{e}^{-\frac{\alpha N}{4} \mu^{2}} q \sinh \pi q\left|\Gamma\left(-\frac{\mu}{2}+i \frac{q}{2}\right)\right|^{2}\left(\frac{p}{\alpha}\right)^{\mu / 2} K_{i q}\left(2 \sqrt{\frac{p}{\alpha}}\right)
$$


b) case $\mu>0$

We choose the contour $\left(\mathcal{C}_{2}\right)$ drawn in figure 2. As in the case $\mu<0$, the integral along the semicircle at infinity vanishes. But now, besides the integral of $f$ along the line $\operatorname{Re}(\mathrm{s})=\mu / 2$, we have to take into account the poles of $\Gamma(-s)$ that are to the left of this line and the poles of $\Gamma(\mu-s)$ that are to the right of this line (see Fig. 2)

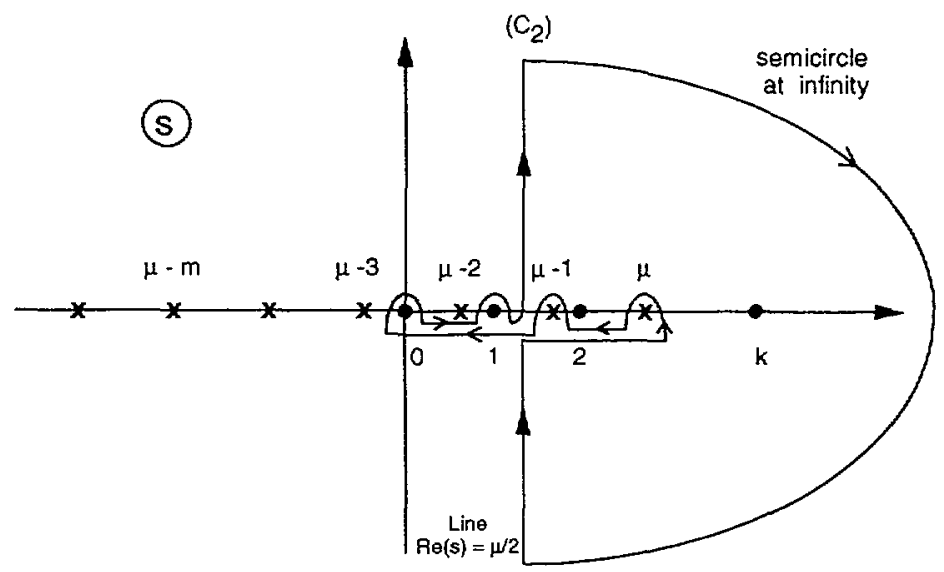

Fig. 2. - Contour $\mathrm{C}_{2}$ for the case $\mu>0$.

$$
\begin{aligned}
\sum_{k=0}^{\infty} u_{k}(x) & =\frac{1}{2 \pi i} \int_{\frac{\mu}{2}-i \infty}^{\frac{\mu}{2}+i \infty} f(s) \mathrm{d} s-\sum_{0 \leq k<\frac{\mu}{2}} \operatorname{Res}(f ; k)+\sum_{0 \leq k<\frac{\mu}{2}} \operatorname{Res}(f ; \mu-k) \\
& =\frac{i}{4 \pi} \int_{-\infty}^{+\infty} \mathrm{d} q q \mathrm{e}^{2 q \sqrt{\alpha N} x}\left|\Gamma\left(-\frac{\mu}{2}+i \frac{q}{2}\right)\right|^{2} I_{2 q}\left(2 \sqrt{\frac{p}{\alpha}}\right) \\
& +\sum_{0 \leq k<\frac{\mu}{2}}\left[\mathrm{e}^{(2 k-\mu) \sqrt{\alpha N} x}-\mathrm{e}^{(\mu-2 k) \sqrt{\alpha N} x}\right] \frac{(-1)^{k}}{k !}(2 k-\mu) \Gamma(k-\mu) I_{2 k-\mu}\left(2 \sqrt{\frac{p}{\alpha}}\right) .
\end{aligned}
$$

After integration over $x$, we recover equation (5.6) for the generating function

$$
\begin{aligned}
\phi(p, N) & =\frac{\mathrm{e}^{-\frac{\alpha N}{4} \mu^{2}}}{2 \pi^{2}} \int_{0}^{\infty} \mathrm{d} q \mathrm{e}^{-\frac{\alpha N}{4} q^{2}} q \sinh \pi q\left|\Gamma\left(-\frac{\mu}{2}+i \frac{q}{2}\right)\right|^{2}\left(\frac{p}{\alpha}\right)^{\mu / 2} K_{2 q}\left(2 \sqrt{\frac{p}{\alpha}}\right) \\
& +\sum_{0 \leq k<\frac{\mu}{2}} \mathrm{e}^{-\alpha N k(\mu-k)} \frac{2(\mu-2 k)}{k ! \Gamma(1+\mu-k)}\left(\frac{p}{\alpha}\right)^{\mu / 2} K_{\mu-2 k}\left(2 \sqrt{\frac{p}{\alpha}}\right)
\end{aligned}
$$




\section{Current between two fixed concentrations.}

When the quenched random force $\{F(x)\}$ is a Gaussian white noise, from the probability density $\psi(\tau, N)$ of the random variable $\tau(N)$, using equation (1.11) we can study some statistical properties of the current $J(N)$. In particular, we will discuss below the behaviour of the average current $\langle J(N)\rangle$ as a function of the drift and of the length of the chain. We also consider the whole distribution of the current in the case $P_{N}=0$.

6.1 AVERAge CURRENT. - The following identities

$$
\left\{\begin{array}{c}
\left\langle\frac{1}{\tau(N)}\right\rangle=\int_{0}^{\infty}\left\langle\mathrm{e}^{-p \tau(N)}>\mathrm{d} p\right. \\
\left\langle\ln (\alpha \tau(N))>=\int_{0}^{\infty} \frac{\left.\mathrm{e}^{-p}-<\mathrm{e}^{-p \alpha \tau(N)}\right\rangle}{p} \mathrm{~d} p\right.
\end{array}\right.
$$

together with the expression of the generating function $\phi(p, N) \equiv\left\langle\mathrm{e}^{-p \tau(N)}\right\rangle(\mathrm{Eq}$. (5.6)) allow us to compute the average current for $\mu \geq 0$ for arbitrary concentrations $\left(P_{0}, P_{N}\right)$ at the two end points

$$
\begin{aligned}
\frac{\langle J(N)>}{\alpha D_{0}} & =P_{0} \mu+\left(P_{0}-P_{N}\right)\left[\sum_{1 \leq n<\frac{\mu}{2}}(\mu-2 n) \mathrm{e}^{-\alpha N n(\mu-n)}\right. \\
& \left.+\frac{1}{2} \int_{0}^{\infty} \mathrm{d} s \mathrm{e}^{-\frac{\alpha N}{4}\left(\mu^{2}+s^{2}\right)} \frac{s \sinh \pi s}{\cosh \pi s-\cos \pi \mu}\right]
\end{aligned}
$$

Let us now discuss some limiting cases.

* For Sinai case $F_{0}=0(\mu=0)$, the average current is proportional to the difference of the end points concentrations according to :

$$
<J(N)>=\frac{2 \alpha}{\pi^{2}}\left(P_{0}-P_{N}\right) \int_{0}^{\infty} d t \mathrm{e}^{-\frac{\alpha N}{\pi^{2}} t^{2}} t \operatorname{coth} t
$$

which is in agreement with Oshanin et al.'s result in the case $P_{N}=0$.

* Limit of vanishing disorder $\sigma \rightarrow 0$

$$
\text { In this limit }\left\{\begin{array}{l}
\mu=\frac{2 F_{0}}{\sigma \beta} \rightarrow \infty \\
\alpha=\frac{\sigma \beta^{2}}{2} \rightarrow 0
\end{array} \text { with } \mu \alpha=F_{0} \beta\right. \text { constant, the spectrum is purely discrete, }
$$

and the average current therefore reads

$$
\begin{gathered}
<J(N)>\underset{\sigma \rightarrow 0}{\rightarrow} D_{0} P_{0} F_{0} \beta+D_{0}\left(P_{0}-P_{N}\right) F_{0} \beta \sum_{n=1}^{\infty} \mathrm{e}^{-N F_{0} \beta n} \\
=D_{0} F_{0} \beta\left[P_{0}+\frac{P_{0}-P_{N}}{\mathrm{e}^{\beta F_{0} N}-1}\right]
\end{gathered}
$$

We recover the deterministic current $J_{\text {det }}(N)$ resulting from a constant force $F_{0}$, since

$$
\tau_{\text {det }}(N)=\int_{0}^{N} \mathrm{~d} x \mathrm{e}^{-\beta F_{0} x}=\frac{1-\mathrm{e}^{-\beta F_{0} N}}{\beta F_{0}}
$$


* Limit of a very long chain $N \rightarrow \infty$

As soon as $\mu>0$, the average current remains finite in the limit $N \rightarrow \infty$

$$
<J(N)>\underset{N \rightarrow \infty}{\rightarrow} \alpha D_{0} P_{0} \mu
$$

The correction to this asymptotic current for large $N$ will exhibit a transition at $\mu=2$ depending on the existence in the spectrum of a discrete state above the ground state. (See the discussion following Eq. (5.6)). More precisely :

- For $\mu>2$, the relaxation is governed by the exponential of the energy of the discrete state $n=1$

$$
\left\langle J(N)>\underset{N \rightarrow \infty}{\sim} \alpha D_{0}\left[P_{0} \mu+\left(P_{0}-P_{N}\right)(\mu-2) \mathrm{e}^{-\alpha N(\mu-1)}+\cdot\right]\right.
$$

- For $0<\mu<2$, the continuous branch modifies the purely exponential decay of the gap $\mathrm{e}^{-\frac{\alpha N}{4} \mu^{2}}$ by an algebraic factor $N^{-3 / 2}$

$$
\left\langle J(N)>\underset{N \rightarrow \infty}{\sim} \alpha D_{0}\left[P_{0} \mu+\left(P_{0}-P_{N}\right) \frac{\pi^{3 / 2}}{1-\cos \pi \mu} \frac{e^{-\frac{\alpha N}{4} \mu^{2}}}{(\alpha N)^{3 / 2}}+\cdot\right]\right.
$$

- For $\mu=0$, there is no normalizable ground state, therefore the continuous branch makes the current vanish purely algebraically like $N^{-1 / 2}$ (cf. Oshanin et al.)

$$
\left\langle J(N)>\underset{N \rightarrow \infty}{\sim} D_{0}\left(P_{0}-P_{N}\right) \sqrt{\frac{\alpha}{\pi N}}+.\right.
$$

6.2 Probability Density of $J(N)$ For the CAse $P_{N}=0$. - When the end at $x=N$ is a trap described by the boundary condition $P_{N}=0$, the relation between $\tau(N)$ and $J(N)$ becomes very simple

$$
J(N)=\frac{D_{0} P_{0}}{\tau(N)}=\frac{J_{0}}{\alpha \tau(N)} \quad \text { where } \quad J_{0} \equiv \alpha D_{0} P_{0}
$$

Equation (5.5) for the probability density $\psi(\tau, N)$ gives immediately the whole distribution of the current $J(N)$

$$
\begin{aligned}
& \mathcal{P}(J, N)=\psi(\tau, N)\left|\frac{\mathrm{d} \tau}{\mathrm{d} J}\right| \\
& =\frac{1}{J_{0}}\left[\sum_{0 \leq n<\frac{\mu}{2}} \mathrm{e}^{-\alpha N n(\mu-n)} \frac{(-1)^{n}(\mu-2 n)}{\Gamma(1+\mu-n)}\left(\frac{J}{J_{0}}\right)^{\mu-1-n} L_{n}^{\mu-2 n}\left(\frac{J}{J_{0}}\right) \mathrm{e}^{-J / J_{0}}\right. \\
& \left.+\frac{1}{4 \pi^{2}} \int_{0}^{\infty} \mathrm{d} s \mathrm{e}^{-\frac{\alpha N}{4}\left(\mu^{2}+s^{2}\right)} s \sinh \pi s\left|\Gamma\left(-\frac{\mu}{2}+i \frac{s}{2}\right)\right|^{2}\left(\frac{J}{J_{0}}\right)^{\frac{\mu-3}{2}} W_{\frac{1+\mu}{2}, 2 \frac{s}{2}}\left(\frac{J}{J_{0}}\right) \mathrm{e}^{-\frac{J}{2 J_{0}}}\right]
\end{aligned}
$$

The asymptotic law for the current in the limit $N \rightarrow \infty$ therefore reads for $\mu>0$

$$
\mathcal{P}(J, N) \underset{N \rightarrow \infty}{\rightarrow} \mathcal{P}_{\infty}(J)=\frac{1}{J_{0} \Gamma(\mu)}\left(\frac{J}{J_{0}}\right)^{\mu-1} \mathrm{e}^{-\frac{J}{J_{0}}}
$$



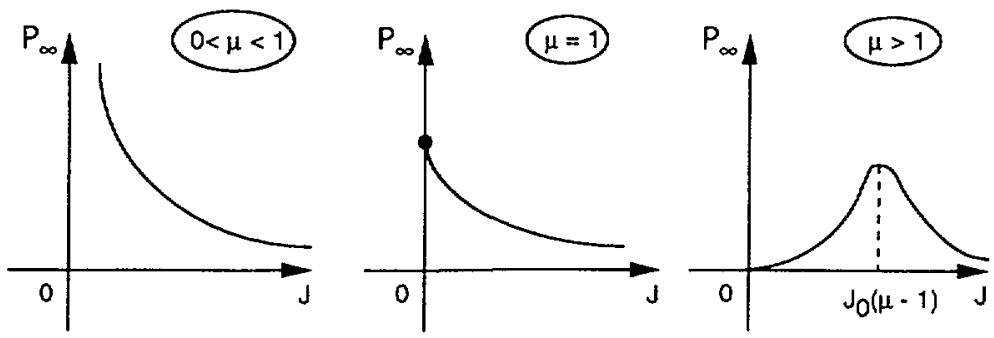

Fig. 3. - Flux distribution for an infinite system. The transition at $\mu=1$ is displayed.

The moment of order $n$ is simply

$$
<J_{\infty}^{n}>=J_{0}^{n} \frac{\Gamma(\mu+n)}{\Gamma(\mu)}=J_{0}^{n} \mu(\mu+1) \cdot(\mu+n-1) .
$$

The curve $\mathcal{P}_{\infty}(J)$ (see Fig. 3) displays a maximum for $\mu>1$. This implies that the most probable value of the asymptotic current $J_{\infty}$ presents a transition at $\mu=1$

$$
J_{\infty}^{*}=\left\{\begin{array}{ccc}
0 & \text { if } & 0<\mu \leq 1 \\
J_{0}(\mu-1) & \text { if } & \mu \geq 1
\end{array}\right.
$$

This transition at $\mu=1$ was already found [3] for the velocity $V$ defined as $V=\lim _{t \rightarrow \infty} \frac{\mathrm{d}}{\mathrm{d} t} \overline{x(t)}$ for each environnement $\{F(x)\}$. It was shown [11] that $V$ was a selfaveraging quantity (4) satisfying

$$
\left\{\begin{array}{ccc}
V=0 & \text { for } & 0<\mu \leq 1 \\
V=\frac{F_{0}}{\gamma}\left(1-\frac{1}{\mu}\right) \equiv \frac{J_{0}}{P_{0}}(\mu-1) & \text { for } & \mu \geq 1
\end{array}\right.
$$

Here the current $J_{\infty}$ is not a self-averaging quantity but its most probable value is simply proportional to the velocity $V$ defined above : $J_{\infty}^{*}=P_{0} V$.

In contrast, the average current $\left\langle J_{\infty}\right\rangle=J_{0} \mu$ does not display any transition at $\mu=1$. As emphasized by Oshanin et al., this average value is supported by atypical realisations of the disorder configurations $\{F(x)\}$.

The Sinai case has to be discussed separately. On a finite chain the behaviour for $J \rightarrow 0$ is given by a log-normal law

$$
\mathcal{P}(J, N) \underset{J \rightarrow 0}{\sim} \frac{1}{2 \sqrt{\pi \alpha N}} \mathrm{e}^{-\frac{1}{4 \alpha N} \ln ^{2}\left(\frac{J}{J_{0}}\right)}
$$

For $J \rightarrow \infty$ one obtains

$$
\mathcal{P}(J, N) \underset{J \rightarrow \infty}{\sim} \frac{1}{\sqrt{\pi \alpha N}} \mathrm{e}^{\frac{\pi^{2}}{4 \alpha N}} \frac{\mathrm{e}^{-J}}{J}
$$

The curve $\mathcal{P}(J, N)$ (see Fig. 4) therefore displays a maximum for a critical value $J_{N}^{*}$. When 


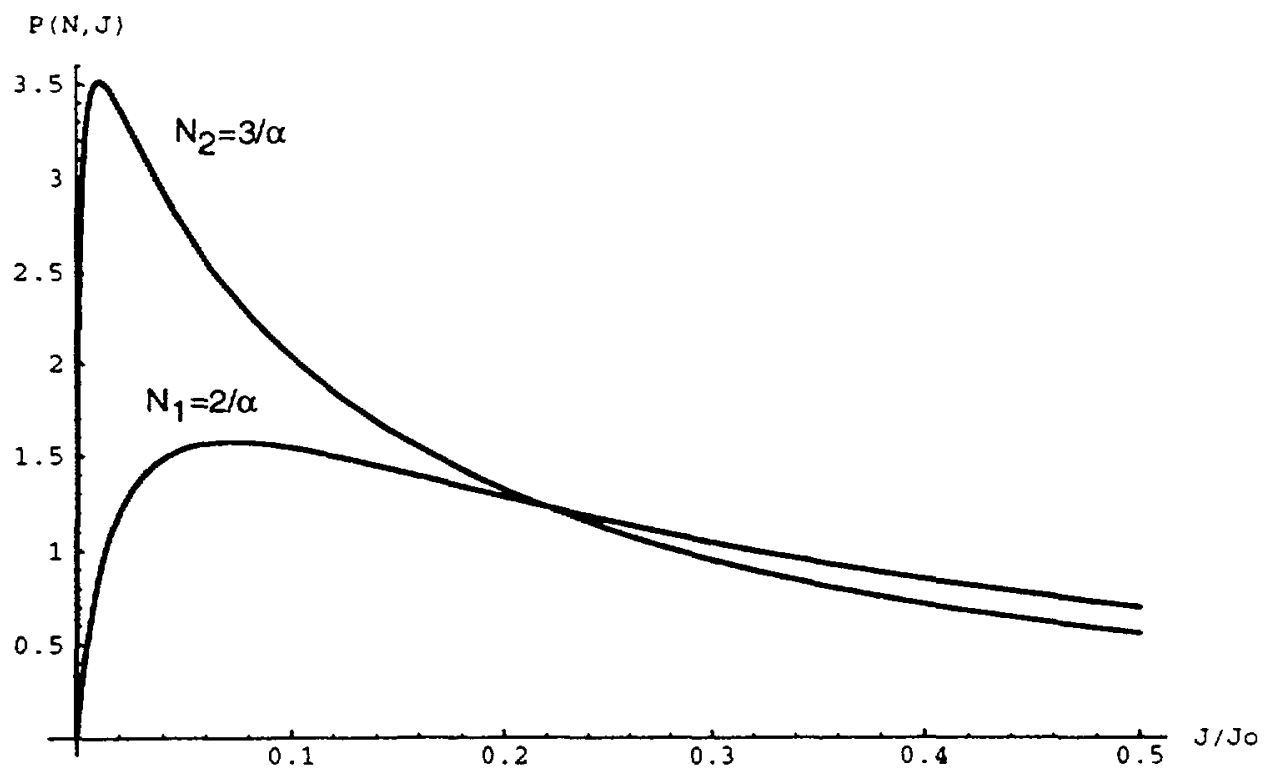

Fig. 4. - Flux distribution in the case $\mu=0$ for two different lenghts $N_{1}=2 / \alpha$ and $N_{2}=3 / \alpha$.

the length $N$ of the system increases, this maximum is pushed towards the origin

$$
\begin{gathered}
J_{N}^{*} \underset{N \rightarrow \infty}{\sim} J_{0} \mathrm{e}^{-2 N \alpha} \\
\mathcal{P}\left(J_{N}^{*}, N\right) \underset{N \rightarrow \infty}{\sim} \frac{1}{2 \sqrt{\pi \alpha N}} \mathrm{e}^{\alpha N}
\end{gathered}
$$

The whole normalisation is therefore concentrated in a region which shrinks to zero in the limit $N \rightarrow \infty$.

\section{Conclusion.}

In this work we have studied the probability distribution of the current $J$ that goes through a one dimensional system of finite length when prescribed concentrations are imposed at its end points. The resulting expressions exhibit many interesting features both as a function of the drift parameter $\mu$ and of the length. We have shown that $\tau \propto 1 / J$ is distributed according to a broad distribution which crucially depends on the drift. In particular, in the Sinai case, this distribution does not tend to a limiting law when the size of the system increases.

In the case $\mu>0$, it is interesting to point out that the same limiting distribution occurs in the context of localization in quantum systems. At the transition between the insulating and conducting regime, conductance fluctuations become very large, therefore the average conductance $\langle g\rangle$ gives a very poor description of the conducting properties of the system. A complete characterisation requires in fact the knowledge of the whole distribution $P(g)$. A perturbative calculation in $2+\epsilon$ dimensions shows that the conductance distribution is characterised by the following cumulants [15]

$$
C_{n}(L)=\left\{\begin{array}{ccc}
\epsilon^{n-2} & \text { for } \quad n \leq n_{0} \\
(L / l)^{\epsilon n^{2}-2 n} & \text { for } \quad n \geq n_{0}
\end{array}\right.
$$


where $n_{0}$ is a large integer of order $1 / \epsilon$ and the size $L$ of the system is much larger than the mean free path $l$. Therefore the large order cumulants diverge when $L \rightarrow \infty$. Since they depend on the mean free path, it a priori suggests that the limiting distribution, if it exists, will depend on $l$ and therefore will not have a universal behavior. This point was carefully investigated in [15]. It was shown that the conductance distribution approaches a universal function $P^{*}(g)$ when $L \rightarrow \infty$ (in spite of the fact that the cumulant are $l$-dependant). The latter can be computed from the cumulant generating function

$$
G_{L}(\lambda)=\sum_{n=0}^{\infty} \frac{(-\lambda)^{n}}{n !} C_{n}(L)
$$

which has the limiting form

$$
G^{*}(\lambda)=\frac{2^{p+1}}{\Gamma(2 p)} p^{2} \lambda^{p} K_{2 p}(\sqrt{8 \lambda})
$$

where $p=1 / \epsilon$. Comparing with (4.5) and (4.7), we see that the following correspondence holds $\mu=2 / \epsilon$ and $N=\frac{2}{\mu \alpha} \ln \left(\frac{L}{l}\right)$. In both systems, the higher moments diverge with the length of the system. We find very puzzling that these two problems give rise to very similar behaviour. This is all the more surprising as they are of a very different nature, since one is classical and the other purely quantum.

Another point that deserves attention is the fact that the determination of the flux distribution involves a differential equation that also arises in the context of stochastic multiplicative processes. Whether or not this is a concidence is an open question. As a final remark, let us point out that the straightforwardness of the functional derivation suggests that an extension in higher dimensions should be considered seriously. Other functionals of a Brownian process that appear for instance in the context of localization can probably be handled in a similar way.

\section{Acknowledgements.}

We have the pleasure to thank Dr. G. Oshanin and Prof. M. Moreau for communicating us their results prior to publication and for an interesting discussion. We also thank Dr. J.P. Bouchaud for his comments and remarks and Dr J. Desbois for his generous help with some numerical calculations.

\section{Appendix A}

We present in this appendix the analytical solution given by Schenzle [9] of the equation $\frac{\partial P}{\partial t}=\frac{\partial^{2}}{\partial x^{2}}\left(x^{2} P\right)-\frac{\partial}{\partial x}\left[(2 \mu+1) x-x^{3}\right] P$ with the initial condition $P(x, t) \underset{t \rightarrow 0^{+}}{\rightarrow} \delta\left(x-x_{0}\right)$.

It is convenient to set $P(x, t)=\sqrt{P_{0}(x)} f(x, t)$ where $P_{0}(x)=\frac{2^{1-\mu}}{\Gamma(\mu)} x^{2 \mu-1} \mathrm{e}^{-\frac{x^{2}}{2}}$.

Since the evolution of $f(x, t)$ is governed by a self-adjoint operator we can use its elgenfunctions to contruct an orthonormal basis $\left(f_{n}\right)$ with corresponding eigenvalues $\left(\lambda_{n}\right)$. The general solution $f(x, t)$ can thus be expanded in the following form

$$
f(x, t)=\sum_{n} c_{n} f_{n}(x) e^{-\lambda_{n} t}
$$


The general solution for $P(x, t)$ is thus given by

$$
P(x, t)=\sum_{n} c_{n} P_{n}(x) \mathrm{e}^{-\lambda_{n} t} \quad \text { where } \quad P_{n}(x)=\sqrt{P_{0}(x)} f_{n}(x, t) .
$$

The orthogonality and closure relations read

$$
\left\{\begin{array}{l}
\int \mathrm{d} x f_{n}(x) f_{m}(x)=\delta_{n, m}=\int \mathrm{d} x \frac{P_{n}(x) P_{m}(x)}{P_{0}(x)} \\
\sum_{n} f_{n}(x) f_{n}\left(x_{0}\right)=\delta\left(x-x_{0}\right) \Rightarrow \sum_{n} P_{n}(x) P_{n}\left(x_{0}\right)=P_{0}\left(x_{0}\right) \delta\left(x-x_{0}\right) .
\end{array}\right.
$$

The initial condition $P(x, t) \underset{t \rightarrow 0^{+}}{\rightarrow} \delta\left(x-x_{0}\right)$ determines the coefficients $c_{n}=\frac{P_{n}\left(x_{0}\right)}{P_{0}\left(x_{0}\right)}$.

The Green function of the problem therefore reads

$$
P\left(x t \| x_{0} 0\right)=\sum_{n} \frac{P_{n}\left(x_{0}\right)}{P_{0}\left(x_{0}\right)} P_{n}(x) \mathrm{e}^{-\lambda_{n} t}
$$

The spectrum $\left(P_{n}, \lambda_{n}\right)$ given by Schenzle consists of a continuous and a discrete branch

\section{* Continuous branch of the spectrum}

$$
s \geq 0\left\{\begin{array}{l}
\lambda(s)=\mu^{2}+s^{2} \\
P_{s}(x)=\frac{1}{\pi} \sqrt{\frac{2^{1-\mu} s \sinh \pi s\left|\Gamma\left(-\frac{\mu}{2}+i \frac{s}{2}\right)\right|^{2}}{\Gamma(\mu)}} x^{\mu-2} \mathrm{e}^{-\frac{x^{2}}{4}} W_{\frac{1}{2}+\frac{\mu}{2}, i \frac{s}{2}}\left(\frac{x^{2}}{2}\right) .
\end{array}\right.
$$

* Discrete branch of the spectrum

$$
0 \leq n<\frac{\mu}{2}\left\{\begin{array}{l}
\lambda_{n}=4 n(\mu-n) \\
P_{n}(x)=2^{n-\mu+1} \sqrt{\frac{n ! 2(\mu-2 n)}{\Gamma(\mu) \Gamma(\mu+1-n)}} x^{2 \mu-2 n-1} L_{n}^{\mu-2 n}\left(\frac{x^{2}}{2}\right) \mathrm{e}^{-\frac{x^{2}}{4}}
\end{array}\right.
$$

Next we perform the change of variables $\left(t=\alpha N / 4\right.$ and $\left.\alpha \tau=\frac{2}{x^{2}}\right)$ and compute the coefficients we need as

$$
c_{n}=\lim _{x_{0} \rightarrow \infty} \frac{P_{n}\left(x_{0}\right)}{P_{0}\left(x_{0}\right)} \quad \text { and } \quad c_{s}=\lim _{x_{0} \rightarrow \infty} \frac{P_{s}\left(x_{0}\right)}{P_{0}\left(x_{0}\right)}
$$

This gives equation (5.5).

\section{References}

[1] Bouchaud J.P. and Georges A., Phys. Rep. 195 (1990) 127;

Havlin S. and Ben-Avraham D., Adv. Phys. 36 (1987) 695.

[2] Sinai Ya. G., Lecture Notes in Physics, R. Schrader, R. Seiler and D. Uhlenbrock Eds., Vol. 53, (Springer verlag, Berlin 1981); Theory Probab. Appl. 27 (1982) 247.

[3] Derrida B. and Pomeau Y., Phys. Rev. Lett. 48 (1982) 627.

[4] Bouchaud J.P., Comtet A., Georges A. and Le Doussal P., Ann. Phys. 201 (1990) 285; 
Georges A., thèse de doctorat, Université Orsay (1988).

[5] KestenH., Acta Math. 131 (1973) 208.

[6] De Calan C., Luck J.M., Nieuwenhuizen Th. M. and Petritis D., J. Phys. A18 (1985) 501.

[7] Derrida B. and Hilhorst H.J., J. Phys. A16 (1983) 2641.

[8] Weiss G.H. and Rubin R.J., Adv. Chem. Phys. 52 (1983) 363.

[9] Oshanın G., Mogutov A. and Moreau M., J. Stat. Phys. 73 (1993).

[10] Burlatsky S.F., Oshanin G.S., Mogutov A.V. and Moreau M., Phys. Rev. A45 (1992) 6955.

[11] Feller W., An introduction to probability theory and its applications, Vol.2, (Wiley, 1966) p.227.

[12] Schenzle A. and Brand H., Phys. Rev. A20 (1979) 1629.

[13] Graham R. and Schenzle A., Phys. Rev. A25 (1981) 1731.

[14] Aslangul C., Bouchaud J.P., Georges A., Pottier N. and Saint James D., J. Stat. Phys. 55 (1989) 461.

[15] Cohen A. and Shapiro B., Int. J. Mod. Phys. B6 (1992) 1243. 